\title{
Galerkin BEM with direct evaluation of hypersingular integrals
}

\author{
Marc Bonnet \\ Laboratoire de Mècanique des Solides \\ Ecole Polytechnique, 91128 Palaiseau cedex, France \\ bonnet@lms.polytechnique.fr \\ Massimo Guiggiani \\ Dipartimento di Ingegneria Meccanica, Nucleare e della Produzione \\ Università di Pisa, via Diotisalvi 2, 56126 Pisa, Italy \\ guiggiani@ing.unipi.it
}

\begin{abstract}
In this paper a new general algorithm is presented for the direct evaluation of all singular double integrals arising in the 2D Galerkin BEM, including those with hypersingular kernels. A distinguishing feature of the proposed method is that double singular integrals are treated as a whole, that is not as inner integrals followed by outer ones. Therefore, when applied to the symmetric Galerkin BEM, the proposed technique is strictly symmetry preserving. Moreover, a careful analysis of the limiting process is performed which shows that some new free terms may arise.
\end{abstract}

\section{Introduction}

Frank Rizzo in his seminal paper [1], published in 1967, was already well aware of the relevance of strongly singular integrals in BEM. However he probably did not expect that the treatment of strongly singular, and later of hypersingular integrals, could give rise to so many techniques and ideas.

Among others, the direct approach [2]-[7] for the evaluation of element integrals arising from the discretization of strongly singular and hypersingular boundary integral equations is now well established for the collocation BEM and widely used. Initially it was developed for strongly singular integrals [2, 3]. Its extension to hypersingular integrals was first published in [4], a paper originated at Iowa State University under the supervision of Frank Rizzo.

In this paper we will briefly report about the extension of the direct approach to the symmetric Galerkin BEM (SGBEM). A complete treatment can be found in the recent paper [8].

When SGBEM was first formulated [9, 10] very little attention was paid to the limiting process which has necessarily to be performed to obtain a boundary integral equation (BIE) with singular kernels. Since then the treatment of singular integrals in SGBEM has received considerable attention.

In most cases the basic idea has been to reduce the order of singularity of the kernel functions thus making the limiting process trivial. In [11] simple solutions were employed, whereas in [12] a procedure based on Stokes theorem (or integration by 
parts in 2D, as in $[13,14])$ was developed. Regularization via integration by parts has been also presented in [15] for Kirchhoff plates. Another approach for the evaluation of singular integrals, often called limit to the boundary, was presented for SGBEM by Balakrishna et al. in $[16,17]$. Recent developments on this technique can be found in [18]. SGBEM formulations based on the so called finite part integrals have been also developed. In [19, 20] they are evaluated numerically, while in [21] an analytical evaluation is pursued.

A method specifically designed to compute integrals with strongly singular kernels for the (unsymmetric) Galerkin BEM in 3D originated in [22] and was enhanced in [23]. It takes advantage of certain symmetry property exhibited by all Cauchy singular kernel functions, even when mapped onto the parameter space. To apply it in the SGBEM, that is to hypersingular kernels, an analytic regularization is therefore necessary, as shown in [26] and [27], where simple solutions and Stokes theorem are employed, respectively.

The present paper deals with the extension of the direct approach to hypersingular boundary integral equations in weighted form, like those arising in the SGBEM. The extension is by no means trivial since the Galerkin BEM involves double element integrals. The algorithm presented here deals with both the coincident and adjacent cases, for 2D problems. Indeed it appears that they must be considered together to allow cancellation of potentially unbounded terms.

Double integrals are considered as a whole (i.e. not as inner singular integrals followed by outer nonsingular ones, like e.g. in Ref. [26]) through the introduction of suitable coordinate transformations in the two-dimensional space of intrinsic coordinates. The proposed algorithm is in particular applicable to the symmetric Galerkin BEM (SGBEM) and is devised so as to define in that case a perfectly symmetric integration procedure, even when the numerical quadrature is not exact.

In line with previous works on the direct approach, the limiting form of the weighted integral identity must be derived as a small neighborhood of the singular point vanishes, and this is done, again, after discretization. (Of course, the finite part of divergent integrals is never employed.)

It is also shown that a new kind of finite free terms arises. It should be noted that, like in the collocation BEM, these free terms are related to the way the limiting process is performed, not to the algorithm eventually adopted for the evaluation of singular integrals. In the present case the limit is carried out on the weighted integral identity. Apparently, an analysis of the free terms arising in the SGBEM based on the vanishing exclusion neighbourhood was so far missing.

\section{Weighted integral statements}

We deal with two-dimensional problems associated with linear homogeneous elliptic field equations, among which the Laplace equation $\nabla^{2} \varphi=0$ is the simplest and most common. Let $\Omega$ be a $2 \mathrm{D}$ bounded domain with boundary $\Gamma=\partial \Omega$ (possibly with a finite number of corners). By $\boldsymbol{n}(\boldsymbol{x})$ and $\boldsymbol{t}(\boldsymbol{x})$ we indicate, respectively, the normal and tangent unit vectors at the generic point $\boldsymbol{x} \in \Gamma$ (Figure 1). Cartesian coordinates are used relative to an orthonormal frame $\left(\boldsymbol{e}_{1}, \boldsymbol{e}_{2}\right)$, so that e.g. $\boldsymbol{x}=x_{i} \boldsymbol{e}_{i}$. The starting 


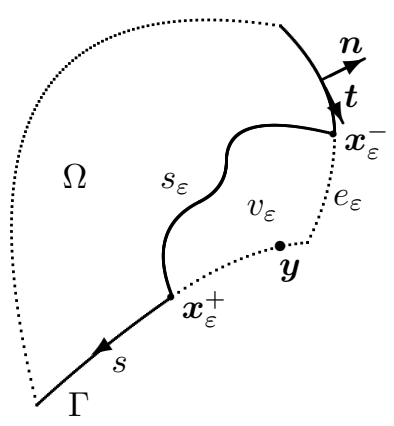

Figure 1: Exclusion of the singular point $\boldsymbol{y}$ by a vanishing neighbourhood $v_{\varepsilon}$.

statement is the classical 3rd Green identity for the density function $\varphi$ and its normal derivative $q=\partial \varphi / \partial n$ on the punctured domain $\Omega_{\varepsilon}=\Omega-v_{\varepsilon}$, with boundary $\Gamma-e_{\varepsilon}+s_{\varepsilon}$ (Figure 1)

$$
\int_{\Gamma-e_{\varepsilon}+s_{\varepsilon}}[T(\boldsymbol{y}, \boldsymbol{x}) \varphi(\boldsymbol{x})-G(\boldsymbol{y}, \boldsymbol{x}) q(\boldsymbol{x})] \mathrm{d} s_{x}=0,
$$

where $\boldsymbol{y} \in \Gamma$ is the singular point, $G(\boldsymbol{y}, \boldsymbol{x})$ is a fundamental solution and

$$
T(\boldsymbol{y}, \boldsymbol{x})=\frac{\partial G(\boldsymbol{y}, \boldsymbol{x})}{\partial n(\boldsymbol{x})}=\frac{\partial G(\boldsymbol{y}, \boldsymbol{x})}{\partial x_{j}} n_{j}(\boldsymbol{x})
$$

As usual, $\varepsilon>0$ is a fixed length that controls the size of $v_{\varepsilon}$ and hence of $s_{\varepsilon}$ and $e_{\varepsilon}$.

If we differentiate $G$ and $T$ with respect to any Cartesian coordinate $y_{i}$ of the singular point and multiply by $n_{i}(\boldsymbol{y})$, we obtain a new pair of (more singular) kernel functions

$$
\begin{aligned}
& \frac{\partial G(\boldsymbol{y}, \boldsymbol{x})}{\partial y_{i}} n_{i}(\boldsymbol{y})=W(\boldsymbol{y}, \boldsymbol{x})=T(\boldsymbol{x}, \boldsymbol{y}) \\
& \frac{\partial T(\boldsymbol{y}, \boldsymbol{x})}{\partial y_{i}} n_{i}(\boldsymbol{y})=V(\boldsymbol{y}, \boldsymbol{x})=V_{i j}(\boldsymbol{y}, \boldsymbol{x}) n_{i}(\boldsymbol{y}) n_{j}(\boldsymbol{x}),
\end{aligned}
$$

which can be combined, exactly like in equation (1), in a hypersingular boundary integral identity

$$
\int_{\Gamma-e_{\varepsilon}+s_{\varepsilon}}[V(\boldsymbol{y}, \boldsymbol{x}) \varphi(\boldsymbol{x})-W(\boldsymbol{y}, \boldsymbol{x}) q(\boldsymbol{x})] \mathrm{d} s_{x}=0 .
$$

It should be noted that for the normal $\boldsymbol{n}(\boldsymbol{y})$ to be uniquely defined, the boundary $\Gamma$ at $\boldsymbol{y}$ must be smooth, but this is standard in the Galerkin BEM since $\boldsymbol{y}$ is always located within a boundary element, even when $\Gamma$ has corners.

It is useful to recall that the fundamental solutions are singular at $\boldsymbol{y}=\boldsymbol{x}$

$$
\begin{aligned}
& G(\boldsymbol{y}, \boldsymbol{x})=O(\ln r) \quad T(\boldsymbol{y}, \boldsymbol{x})=O\left(r^{-1}\right) \\
& W(\boldsymbol{y}, \boldsymbol{x})=O\left(r^{-1}\right) \quad V(\boldsymbol{y}, \boldsymbol{x})=O\left(r^{-2}\right)
\end{aligned}
$$


(where $\boldsymbol{r}=\boldsymbol{x}-\boldsymbol{y}$ and $r=|\boldsymbol{r}|$ ), and that they possess the following symmetry properties:

$$
G(\boldsymbol{y}, \boldsymbol{x})=G(\boldsymbol{x}, \boldsymbol{y}) \quad T(\boldsymbol{y}, \boldsymbol{x})=W(\boldsymbol{x}, \boldsymbol{y}) \quad V(\boldsymbol{y}, \boldsymbol{x})=V(\boldsymbol{x}, \boldsymbol{y})
$$

In this paper, the hypersingular kernel $V_{i j}(\boldsymbol{y}, \boldsymbol{x})$ is assumed to have the following, quite general, form:

$$
V_{i j}(\boldsymbol{y}, \boldsymbol{x})=V_{i j}(\boldsymbol{e}, r, \boldsymbol{y})=\frac{1}{r^{2}} \mathcal{V}_{i j}(\boldsymbol{e}, r, \boldsymbol{y}) \quad(\boldsymbol{y} \neq \boldsymbol{x})
$$

where $\mathcal{V}_{i j}$ is bounded in the limiting case $\boldsymbol{x}=\boldsymbol{y}$, i.e. $r=0$, and the unit vector $\boldsymbol{e}$ is defined as $\boldsymbol{r} / r$. In addition, the symmetry property (6) implies that the nonsingular factor $\mathcal{V}_{i j}(\boldsymbol{e}, r, \boldsymbol{y})$ must satisfy:

$$
\mathcal{V}_{i j}(\boldsymbol{e}, r, \boldsymbol{y})=\mathcal{V}_{j i}(-\boldsymbol{e}, r, \boldsymbol{x})
$$

The nonsingular factor $\mathcal{V}_{i j}$ is bounded at $\boldsymbol{x}=\boldsymbol{y}$. If $\boldsymbol{y}$ lies within a region having homogeneous constitutive property, the singular behavior is that of the full-space fundamental solution, e.g.

$$
\mathcal{V}_{i j}(\boldsymbol{e}, 0, \boldsymbol{y})=\frac{1}{2 \pi}\left[\delta_{i j}-2 e_{i} e_{j}\right]
$$

for the Laplace equation.

To facilitate generalizations (e.g. to anisotropic media and elasticity), the nonsingular factor is assumed in this paper to fulfill the requirement

$$
\left.\frac{\partial}{\partial r} \mathcal{V}_{i j}(\boldsymbol{e}, r, \boldsymbol{y})\right|_{r=0}=0
$$

Let $\psi(\boldsymbol{y})$ be a function defined on $\Gamma$. Before taking the limit for $\varepsilon \rightarrow 0$, we can use $\psi(\boldsymbol{y})$ to weigh the former integral identities (1) and (4) as typically done in the Galerkin BEM. For the hypersingular identity (4) we have

$$
\int_{\Gamma} \psi(\boldsymbol{y})\left\{\int_{\Gamma-e_{\varepsilon}+s_{\varepsilon}}[V(\boldsymbol{y}, \boldsymbol{x}) \varphi(\boldsymbol{x})-W(\boldsymbol{y}, \boldsymbol{x}) q(\boldsymbol{x})] \mathrm{d} s_{x}\right\} \mathrm{d} s_{y}=0,
$$

or

$$
\begin{aligned}
\int_{\Gamma} \psi(\boldsymbol{y})\left\{\int_{\Gamma-e_{\varepsilon}}[\right. & V(\boldsymbol{y}, \boldsymbol{x}) \varphi(\boldsymbol{x})-W(\boldsymbol{y}, \boldsymbol{x}) q(\boldsymbol{x})] \mathrm{d} s_{x} \\
& \left.+\int_{s_{\varepsilon}}[V(\boldsymbol{y}, \boldsymbol{x}) \varphi(\boldsymbol{x})-W(\boldsymbol{y}, \boldsymbol{x}) q(\boldsymbol{x})] \mathrm{d} s_{x}\right\} \mathrm{d} s_{y}=0 .
\end{aligned}
$$

This kind of weighted hypersingular integral identities are useful, for instance, in the symmetric Galerkin BEM. As it will be shown, the algorithm will be developed consistently with the assumption that the external integration precedes the limiting process.

As customary, the density function $\varphi$ is assumed to be $C^{(1, \alpha)}$ at $\boldsymbol{y}$, that is

$$
\begin{array}{rlrl}
\varphi(\boldsymbol{x}) & =\varphi(\boldsymbol{y})+\boldsymbol{\nabla} \varphi(\boldsymbol{y}) \cdot(\boldsymbol{x}-\boldsymbol{y})+O\left(r^{1+\alpha}\right), & \\
\boldsymbol{\nabla} \varphi(\boldsymbol{x}) & =\boldsymbol{\nabla} \varphi(\boldsymbol{y})+O\left(r^{\alpha}\right),
\end{array}
$$


Through the usual addition and subtraction of the above expansions in the integral on $s_{\varepsilon}$ and rearranging terms in the integral identity (12) we obtain

$$
\begin{aligned}
0=\int_{\Gamma} \psi & (\boldsymbol{y})\left\{\int_{\Gamma-e_{\varepsilon}}[V(\boldsymbol{y}, \boldsymbol{x}) \varphi(\boldsymbol{x})-W(\boldsymbol{y}, \boldsymbol{x}) q(\boldsymbol{x})] \mathrm{d} s_{x}+\varphi(\boldsymbol{y}) \int_{s_{\varepsilon}} V(\boldsymbol{y}, \boldsymbol{x}) \mathrm{d} s_{x}\right. \\
& +\nabla \varphi(\boldsymbol{y}) \cdot\left[\int_{s_{\varepsilon}}\{(\boldsymbol{x}-\boldsymbol{y}) V(\boldsymbol{y}, \boldsymbol{x})-\boldsymbol{n}(\boldsymbol{x}) W(\boldsymbol{y}, \boldsymbol{x})\} \mathrm{d} s_{x}\right] \\
& +\int_{s_{\varepsilon}} V(\boldsymbol{y}, \boldsymbol{x})[\varphi(\boldsymbol{x})-\varphi(\boldsymbol{y})-\boldsymbol{\nabla} \varphi(\boldsymbol{y}) \cdot(\boldsymbol{x}-\boldsymbol{y})] \mathrm{d} s_{x} \\
& \left.-\int_{s_{\varepsilon}} W(\boldsymbol{y}, \boldsymbol{x})[\boldsymbol{\nabla} \varphi(\boldsymbol{x})-\boldsymbol{\nabla} \varphi(\boldsymbol{y})] \cdot \boldsymbol{n}(\boldsymbol{x}) \mathrm{d} s_{x}\right\} \mathrm{d} s_{y}
\end{aligned}
$$

which is more suitable for the subsequent direct evaluation of singular integrals and for the computation of free terms.

We seek the limiting form of the weighted equation (14) as $\varepsilon \rightarrow 0$. In principle any shape may be used for $v_{\varepsilon}$, since the overall result will not depend on this shape. Like for the direct approach in collocation BEM, selecting a circular shape is found to facilitate the analysis and is therefore assumed in the remainder of this paper.

The last two integrals on $s_{\varepsilon}$ in equation (14) are $O\left(\varepsilon^{\alpha}\right)$ by virtue of assumption (13) and thus vanish in the limit for $\varepsilon \rightarrow 0$. As it will be shown in Section 6 , the first integral on $s_{\varepsilon}$ in equation (14) gives rise to an unbounded $O\left(\varepsilon^{-1}\right)$ term and, when integrated in the Galerkin BEM, a (somewhat unexpected) bounded free term. The second integral on $s_{\varepsilon}$ in (14) yields a bounded free term $\boldsymbol{c}(\boldsymbol{y})$ in the limit for $\varepsilon \rightarrow 0$

$$
\boldsymbol{c}(\boldsymbol{y})=\lim _{\varepsilon \rightarrow 0} \int_{s_{\varepsilon}}\{(\boldsymbol{x}-\boldsymbol{y}) V(\boldsymbol{y}, \boldsymbol{x})-\boldsymbol{n}(\boldsymbol{x}) W(\boldsymbol{y}, \boldsymbol{x})\} \mathrm{d} s_{x}
$$

which occurs also in the direct approach for the collocation BEM in the hypersingular case, see e.g. [5].

Taking into account the above remarks in (14), the direct Galerkin BEM formulation with hypersingular kernels (like required in the SGBEM) is sought as the limiting form as $\varepsilon \rightarrow 0$ of the weighted identity

$$
\begin{aligned}
0=\int_{\Gamma} \psi(\boldsymbol{y})\{\boldsymbol{\nabla} & \varphi(\boldsymbol{y}) \cdot \boldsymbol{c}(\boldsymbol{y})+\varphi(\boldsymbol{y}) \int_{s_{\varepsilon}} V(\boldsymbol{y}, \boldsymbol{x}) \mathrm{d} s_{x} \\
& \left.+\int_{\Gamma-e_{\varepsilon}}\{V(\boldsymbol{y}, \boldsymbol{x}) \varphi(\boldsymbol{x})-W(\boldsymbol{y}, \boldsymbol{x}) q(\boldsymbol{x})\} \mathrm{d} s_{x}\right\} \mathrm{d} s_{y}+O(\varepsilon)
\end{aligned}
$$

where $O(\varepsilon)$ accounts for all vanishing contributions. It should be noted that within curly braces we have precisely the hypersingular boundary integral equation as obtained for the collocation BEM in $[4,5,6]$, although now the limiting process will wait till after the outer integration is performed. Moreover, the $C^{1, \alpha}$ smoothness assumption for $\varphi$, a standard requirement for the hypersingular collocation BEM, is invoked here mainly to dispose easily of some of the integrals over $s_{\varepsilon}$. Indeed, as a result of the analysis conducted in the following sections, the limiting form as $\varepsilon \rightarrow 0$ of the weighted 
identity (16) will appear to require only the weaker $C^{0, \alpha}$ smoothness assumption for $\varphi$, which is consistent with other published treatments of the SGBEM.

Up to this point everything is pretty classical. New ideas are introduced in the next sections, towards the goal of developing a technique for the direct evaluation of singular double integrals, along with further treatment of the free terms.

\section{Double integration: preliminary definitions}

Let the boundary $\Gamma$ be modeled by (curvilinear) boundary elements and let $\boldsymbol{y} \in E$ and $\boldsymbol{x} \in E^{\prime}$, with $E$ and $E^{\prime}$ being two such boundary elements. Obviously, this implies that $\Gamma$ is smooth within each element.

In the Galerkin BEM (regardless of its symmetry) we have to deal with double integrals on Cartesian products $E \times E^{\prime}$ of elements. Typically, the weight function $\psi$ has limited support and $E$ belongs to it. If the two elements are disjoint, that is, do not share a common endpoint, the double integration

$$
\int_{E} \int_{E^{\prime}}\{\cdots\} \mathrm{d} s_{x} \mathrm{~d} s_{y}
$$

is performed using ordinary means since the integrand is continuous. On the other hand, singularities in the integrand function arise either when the two elements share one common endpoint (i.e., they are adjacent), or when they are coincident (i.e., $E=$ $\left.E^{\prime}\right)$. In these cases, according to the starting identity (16), the appropriate setting is

$$
\int_{E} \int_{E^{\prime}(\boldsymbol{y}, \varepsilon)}\{\cdots\} \mathrm{d} s_{x} \mathrm{~d} s_{y} .
$$

where

$$
E^{\prime}(\boldsymbol{y}, \varepsilon)=\left\{\boldsymbol{x} \in E^{\prime}:|\boldsymbol{x}-\boldsymbol{y}| \geq \varepsilon, \text { with } \boldsymbol{y} \in E\right\}=E^{\prime} \backslash e_{\varepsilon}(\boldsymbol{y}),
$$

In fact, one has $E^{\prime}(\boldsymbol{y}, \varepsilon)=E^{\prime}$ in the disjoint case (17) and for sufficiently small $\varepsilon$, which is thus included in this setting.

Each geometric boundary element is analytically defined by means of (usually polynomial) parametric equations. A point $\boldsymbol{x}$ of $E^{\prime}$ is typically given by

$$
\boldsymbol{x}=\boldsymbol{x}(\xi)=\sum_{p=1}^{N_{e}} N_{p}(\xi) \tilde{\boldsymbol{x}}^{p}
$$

where $\xi \in[-1,1]$ is the parameter (or intrinsic) coordinate, $N_{p}(\xi)$ are cardinal shape functions and $\tilde{\boldsymbol{x}}$ are the geometric nodes of $E^{\prime}$. Of course, the use of cardinal shape function is just a matter of practical convenience. Any set of parametric equations for $\boldsymbol{x}(\xi)$ would fit the purpose.

We will denote by $\boldsymbol{a}(\xi)$ and $\boldsymbol{t}(\xi)$, respectively, the natural and unit tangent vector to the element $E^{\prime}$ at $\boldsymbol{x}(\xi)$

$$
\boldsymbol{a}(\xi)=\frac{\mathrm{d} \boldsymbol{x}}{\mathrm{d} \xi}=\sum_{p=1}^{N_{e}} \frac{\mathrm{d} N_{p}}{\mathrm{~d} \xi}(\xi) \tilde{\boldsymbol{x}}^{p}, \quad \text { so that } \quad \boldsymbol{t}(\xi)=\frac{\boldsymbol{a}(\xi)}{|\boldsymbol{a}(\xi)|}=\frac{\boldsymbol{a}(\xi)}{a(\xi)},
$$


and by $\boldsymbol{m}(\xi)$ and $\boldsymbol{n}(\xi)$ the natural and unit normal vectors:

$$
\boldsymbol{m}(\xi)=\left(\boldsymbol{e}_{1} \wedge \boldsymbol{e}_{2}\right) \wedge \boldsymbol{a}(\xi), \quad \boldsymbol{n}(\xi)=\frac{\boldsymbol{m}(\xi)}{|\boldsymbol{m}(\xi)|}=\frac{\boldsymbol{m}(\xi)}{a(\xi)} .
$$

In terms of components of $\boldsymbol{m}$ and $\boldsymbol{a}$ we have that

$$
m_{1}=-a_{2} \quad m_{2}=a_{1}
$$

Moreover, it should be noted that $a(\xi)=|\boldsymbol{a}(\xi)|$ is the Jacobian. In fact

$$
\boldsymbol{n}(\boldsymbol{x}) \mathrm{d} s_{x}=\boldsymbol{n}(\xi) a(\xi) \mathrm{d} \xi=\boldsymbol{m}(\xi) \mathrm{d} \xi,
$$

where, for simplicity, we adopted the slightly sloppy notation $\boldsymbol{n}(\xi)$ to mean $\boldsymbol{n}(\boldsymbol{x}(\xi))$. We also define

$$
\boldsymbol{b}(\xi)=\frac{\mathrm{d} \boldsymbol{a}}{\mathrm{d} \xi}=\frac{\mathrm{d}^{2} \boldsymbol{x}}{\mathrm{d} \xi^{2}}=\sum_{p=1}^{N_{e}} \frac{\mathrm{d}^{2} N_{p}}{\mathrm{~d} \xi^{2}}(\xi) \tilde{\boldsymbol{x}}^{p} .
$$

Similarly to equation (20), we have for the singular point $\boldsymbol{y} \in E$

$$
\boldsymbol{y}=\boldsymbol{y}(\eta)=\sum_{p=1}^{N_{e}} N_{p}(\eta) \tilde{\boldsymbol{y}}^{p}
$$

with $-1 \leq \eta \leq 1$. Obviously, $\tilde{\boldsymbol{y}}^{p}$ are the geometric nodes of $E$. In general, the distance vector $\boldsymbol{r}$ between the points $\boldsymbol{x}$ and $\boldsymbol{y}$ is therefore given by

$$
\boldsymbol{r}=\boldsymbol{x}(\xi)-\boldsymbol{y}(\eta)=\sum_{p=1}^{N_{e}}\left[N_{p}(\xi) \tilde{\boldsymbol{x}}^{p}-N_{p}(\eta) \tilde{\boldsymbol{y}}^{p}\right]
$$

and has modulus $r=|\boldsymbol{r}|$.

\section{Double integration over coincident elements}

Let $\boldsymbol{x}$ and $\boldsymbol{y}$ belong to the same boundary element $E^{\prime}=E$. We will consider the integral with hypersingular kernel (the strongly singular one is just simpler). According to (18) we have

$$
I_{C}=\int_{E} \int_{E(\boldsymbol{y}, \varepsilon)} V_{i j}(\boldsymbol{y}, \boldsymbol{x}) n_{i}(\boldsymbol{y}) n_{j}(\boldsymbol{x}) \psi(\boldsymbol{y}) \varphi(\boldsymbol{x}) \mathrm{d} s_{x} \mathrm{~d} s_{y},
$$

where $E(\boldsymbol{y}, \varepsilon)$ is defined by (19) with $E^{\prime}=E$. Expression (26) for the distance vector $r$ becomes in this case

$$
\boldsymbol{r}=\boldsymbol{x}(\xi)-\boldsymbol{y}(\eta)=\sum_{p=1}^{N_{e}}\left[N_{p}(\xi)-N_{p}(\eta)\right] \tilde{\boldsymbol{y}}^{p},
$$

and $(r=0) \Leftrightarrow(\boldsymbol{x}=\boldsymbol{y}) \Leftrightarrow(\xi=\eta)$. Therefore, in the parameter space we have to integrate over the square $(\eta, \xi) \in[-1,1] \times[-1,1]$ minus a (narrow, non uniform) strip across the $\xi=\eta$ diagonal, as shown in Figure 2. The strip is the image of $E \times e_{\varepsilon}(\boldsymbol{y})$. 


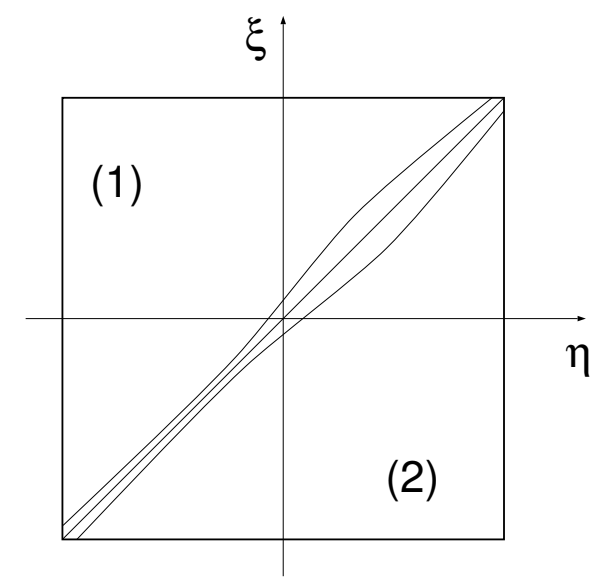

Figure 2: Coincident case: integration domain and exclusion vanishing strip in the parameter space.

\subsection{Coordinate transformations and expansions}

The double integration (27) will be treated by subdividing the square $(\eta, \xi) \in[-1,1] \times$ $[-1,1]$ in the parameter space into two triangular regions, labeled (1) and (2) as shown in Figure 2. In each region a new pair of coordinates $(u, v)$ will be introduced according to the following scheme

$$
\begin{array}{ll}
\text { region (1): } & \left\{\begin{array}{l}
\eta=u-(1+u) v=\eta^{(1)}(u, v) \\
\xi=u+(1-u) v=\xi^{(1)}(u, v)
\end{array}\right. \\
\text { region (2): } & \left\{\begin{array}{l}
\eta=u+(1-u) v=\eta^{(2)}(u, v) \\
\xi=u-(1+u) v=\xi^{(2)}(u, v)
\end{array}\right.
\end{array}
$$

with, in both regions, $u \in[-1,1]$ and $v \in[0,1]$. In some cases the notation $\xi(u, v)$ and $\eta(u, v)$ will be used to refer to (29) and (30).

It should be noted that in both cases if $v=0$ we have $\xi=\eta$, that is, more precisely

$$
\xi^{(i)}(u, 0)=\eta^{(i)}(u, 0)=u \text {. }
$$

Therefore, $v=0$ activates the singularity. Another feature of these coordinate transformations is that $u$ and $v$ vary between fixed values, that is the range of variation of each coordinate does not depend on the other one. Also useful is the relation

$$
\mathrm{d} \xi \mathrm{d} \eta=2(1-v) \mathrm{d} u \mathrm{~d} v .
$$

In terms of the new coordinates $u$ and $v$, the distance vector $\boldsymbol{r}$ defined in (28) becomes

$$
\begin{aligned}
\boldsymbol{r}=\boldsymbol{r}^{(i)}(u, v)=\boldsymbol{x}\left(\xi^{(i)}\right. & (u, v))-\boldsymbol{y}\left(\eta^{(i)}(u, v)\right) \\
& =\sum_{p=1}^{N_{e}}\left[N_{p}\left(\xi^{(i)}(u, v)\right)-N_{p}\left(\eta^{(i)}(u, v)\right)\right] \tilde{\boldsymbol{y}}^{p}
\end{aligned}
$$


We are interested in the Taylor expansion of functions $\boldsymbol{r}^{(i)}(u, v)$ as a function of $v$ and near $v=0$. It is a simple matter to obtain from equation (28) the following more explicit expressions for the derivatives of $\boldsymbol{r}$ appearing in (35)

$$
\begin{aligned}
\left.\frac{\partial \boldsymbol{r}^{(i)}}{\partial v}\right|_{v=0} & =\left[\frac{\mathrm{d} \boldsymbol{x}}{\mathrm{d} \xi} \frac{\partial \xi^{(i)}}{\partial v}-\frac{\mathrm{d} \boldsymbol{y}}{\mathrm{d} \eta} \frac{\partial \eta^{(i)}}{\partial v}\right]_{v=0} \\
& =\sum_{p=1}^{N_{e}}\left[\frac{\mathrm{d} N_{p}}{\mathrm{~d} u}(u)( \pm 1-u)-\frac{\mathrm{d} N_{p}}{\mathrm{~d} u}(u)(\mp 1-u)\right] \tilde{\boldsymbol{y}}^{p} \\
& = \pm 2 \sum_{p=1}^{N_{e}} \frac{\mathrm{d} N_{p}}{\mathrm{~d} u}(u) \tilde{\boldsymbol{y}}^{p}= \pm 2 \boldsymbol{a}(u), \\
\left.\frac{\partial^{2} \boldsymbol{r}^{(i)}}{\partial v^{2}}\right|_{v=0} & =\sum_{p=1}^{N_{e}}\left[\frac{\mathrm{d}^{2} N_{p}}{\mathrm{~d} u^{2}}(u)( \pm 1-u)^{2}-\frac{\mathrm{d}^{2} N_{p}}{\mathrm{~d} u^{2}}(u)(\mp 1-u)^{2}\right] \tilde{\boldsymbol{y}}^{p} \\
& =\mp 4 u \sum_{p=1}^{N_{e}} \frac{\mathrm{d}^{2} N_{p}}{\mathrm{~d} u^{2}}(u) \tilde{\boldsymbol{y}}^{p}=\mp 4 u \boldsymbol{b}(u),
\end{aligned}
$$

where, since $u=\xi=\eta$ when $v=0, \boldsymbol{a}$ and $\boldsymbol{b}$ are precisely the functions defined in equations (21) and (24) (with just $u$ replacing $\xi$ ). The Taylor expansion of $\boldsymbol{r}^{(i)}(u, v)$ readily follows:

$$
\begin{aligned}
\boldsymbol{r}^{(i)}(u, v) & =\boldsymbol{r}^{(i)}(u, 0)+\left.\frac{\partial \boldsymbol{r}^{(i)}}{\partial v}\right|_{v=0} v+\left.\frac{1}{2} \frac{\partial^{2} \boldsymbol{r}^{(i)}}{\partial v^{2}}\right|_{v=0} v^{2}+O\left(v^{3}\right) \\
& =0 \pm 2 \boldsymbol{a}(u) v \mp 2 u \boldsymbol{b}(u) v^{2}+O\left(v^{3}\right) \\
& = \pm 2 v\left[\boldsymbol{a}(u)-u v \boldsymbol{b}(u)+O\left(v^{2}\right)\right] \\
& =v \hat{\boldsymbol{r}}^{(i)}(u, v),
\end{aligned}
$$

The last row defines a continuous function $\hat{\boldsymbol{r}}^{(i)}$

$$
\hat{\boldsymbol{r}}^{(i)}(u, v)= \pm 2 \boldsymbol{a}(u) \mp 2 u v \boldsymbol{b}(u)+O\left(v^{2}\right)
$$

with the property that $\hat{\boldsymbol{r}}^{(i)}(u, 0)= \pm 2 \boldsymbol{a}(u) \neq 0$. Similar definitions (with \pm or $\mp$ ) will be used throughout the paper, where the first sign refers to region (1), and the second one to region (2) (Figure 2).

Similarly, from (36), the distance $r=|\boldsymbol{r}|$ has, the following Taylor expansion

$$
\begin{aligned}
r & =\sqrt{\boldsymbol{r} \cdot \boldsymbol{r}} \\
& =2 v\left[a(u)-u v \frac{\mathrm{d} a}{\mathrm{~d} u}(u)+O\left(v^{2}\right)\right] \\
& =v \hat{r}(u, v),
\end{aligned}
$$

where $a(u)$ is defined by $(21)$ and $\mathrm{d} a / \mathrm{d} u=(\boldsymbol{a}(u) \cdot \boldsymbol{b}(u)) / a(u)$, and having set

$$
\hat{r}(u, v)=|\hat{\boldsymbol{r}}(u, v)|=2 a(u)\left[1-\frac{u v}{a(u)} \frac{\mathrm{d} a}{\mathrm{~d} u}(u)+O\left(v^{2}\right)\right],
$$


while the unit position vector $e$ is such that:

$$
e^{(i)}(u, v)=\frac{\hat{\boldsymbol{r}}^{(i)}(u, v)}{\hat{r}(u, v)}=\boldsymbol{t}(u)+O(v)
$$

The integrand function in equation (27), as a function of $u$ and $v$, becomes

$$
\begin{aligned}
V_{i j}(\boldsymbol{y}, \boldsymbol{x}) n_{i}(\boldsymbol{y}) n_{j}(\boldsymbol{x}) \psi(\boldsymbol{y}) \varphi(\boldsymbol{x}) \mathrm{d} s_{x} \mathrm{~d} s_{y} \\
=\frac{1}{r^{2}} \mathcal{V}_{i j}(\boldsymbol{e}, r, \boldsymbol{y}) m_{i}(\eta) m_{j}(\xi) \psi(\eta) \varphi(\xi) \mathrm{d} \xi \mathrm{d} \eta \\
=\frac{1}{v^{2} \hat{r}^{2}(u, v)} \mathcal{V}_{i j}\left(\boldsymbol{e}^{(i)}(u, v), r(u, v), \boldsymbol{y}\left(\eta^{(i)}(u, v)\right)\right. \\
\quad \times m_{i}\left(\eta^{(i)}(u, v)\right) m_{j}\left(\xi^{(i)}(u, v)\right) \psi\left(\eta^{(i)}(u, v)\right) \varphi\left(\xi^{(i)}(u, v)\right) 2(1-v) \mathrm{d} u \mathrm{~d} v \\
=\frac{1}{v^{2}} \mathcal{F}\left(\xi^{(i)}(u, v), \eta^{(i)}(u, v)\right)(1-v) \mathrm{d} u \mathrm{~d} v \\
=\frac{1}{v^{2}} F^{(i)}(u, v) \mathrm{d} u \mathrm{~d} v .
\end{aligned}
$$

This expression also defines the nonsingular functions $F^{(i)}(u, v)$ and $\mathcal{F}(\xi, \eta)$ (note that $\mathcal{F}$ is the same on regions (1) and (2)). Moreover, $\psi(\eta)$ means $\psi(\boldsymbol{y}(\eta))$ and $\varphi(\xi)$ means $\varphi(\boldsymbol{x}(\xi))$.

The direct algorithm for the evaluation of hypersingular integrals relies on a twoterm Taylor expansion of $F(u, v)$ around $v=0$

$$
F(u, v)=F(u, 0)+\frac{\partial F}{\partial v}(u, 0) v+O\left(v^{2}\right) .
$$

Here, one observes from the definition (40) of $F(u, v)$ and $\mathcal{F}(\xi, \eta)$ that

$$
\begin{aligned}
F^{(i)}(u, 0)=\mathcal{F}\left(\xi^{(i)}(u, 0), \eta^{(i)}\right. & (u, 0))=\mathcal{F}(u, u) \\
& =\frac{1}{2} \mathcal{V}_{i j}(\boldsymbol{t}(u), 0, \boldsymbol{y}(u)) n_{i}(u) n_{j}(u) \psi(u) \varphi(u)
\end{aligned}
$$

and also that

$$
\begin{aligned}
\frac{\partial F^{(i)}}{\partial v} & =\left(\frac{\partial \mathcal{F}}{\partial \xi} \frac{\partial \xi^{(i)}}{\partial v}+\frac{\partial \mathcal{F}}{\partial \eta} \frac{\partial \eta^{(i)}}{\partial v}\right)(1-v)-\mathcal{F}\left(\xi^{(i)}(u, v), \eta^{(i)}(u, v)\right) \\
& =\left(\frac{\partial \mathcal{F}}{\partial \xi}( \pm 1-u)+\frac{\partial \mathcal{F}}{\partial \eta}(\mp 1-u)\right)(1-v)-\mathcal{F}\left(\xi^{(i)}(u, v), \eta^{(i)}(u, v)\right),
\end{aligned}
$$

which means that

$$
\frac{\partial F^{(1)}}{\partial v}+\frac{\partial F^{(2)}}{\partial v}=-2 u\left(\frac{\partial \mathcal{F}}{\partial \xi}+\frac{\partial \mathcal{F}}{\partial \eta}\right)(1-v)-\left[\mathcal{F}\left(\xi^{(1)}, \eta^{(1)}\right)+\mathcal{F}\left(\xi^{(2)}, \eta^{(2)}\right)\right] .
$$


If this expression is evaluated at $v=0$, that is at $\xi=\eta=u$, we obtain

$$
\begin{aligned}
{\left[\frac{\partial F^{(1)}}{\partial v}+\frac{\partial F^{(2)}}{\partial v}\right]_{v=0} } & =-2 u \frac{\mathrm{d}}{\mathrm{d} u}(\mathcal{F}(u, u))-2 \mathcal{F}(u, u) \\
& =-2 \frac{\mathrm{d}}{\mathrm{d} u}(u \mathcal{F}(u, u)) \\
& =-2 \frac{\mathrm{d}}{\mathrm{d} u}(u F(u, 0))
\end{aligned}
$$

where the last step is based on (42). This expression will later prove very useful.

Once the expansion of the singular integrand function has been obtained, we have to consider, as usual in the direct approach, the image in the parameter plane of the exclusion vanishing neighborhood $e_{\varepsilon}(\boldsymbol{y})$ (Figure 1). The boundary of $e_{\varepsilon}(\boldsymbol{y})$ is defined by the condition

$$
r=|\boldsymbol{x}(\xi(u, v))-\boldsymbol{y}(\eta(u, v))|=\varepsilon .
$$

This condition, together with expansion (37) for $r(u, v)$, leads, on each triangular subregion (Figure 2), to

$$
\varepsilon=r(u, v)=v \hat{r}(u, v)=2 v a(u)\left[1-\frac{u v}{a(u)} \frac{\mathrm{d} a}{\mathrm{~d} u}(u)+O\left(v^{2}\right)\right],
$$

which, upon reversion, defines the function

$$
v_{\varepsilon}=\alpha(\varepsilon, u)=\frac{\varepsilon}{2 a(u)}\left[1+\frac{u}{2 a^{2}(u)} \frac{\mathrm{d} a}{\mathrm{~d} u}(u) \varepsilon+O\left(\varepsilon^{2}\right)\right],
$$

The function $\alpha(\varepsilon, u)$ provides, for any given values of $u$ and $\varepsilon$, the value of $v$ corresponding to the boundary of the exclusion strip in Figure 2.

\subsection{Double singular integrals in parametric coordinates}

According to equations (40) and (46), the hypersingular double integral (27) can now be expressed in terms of the parametric coordinates $u$ and $v$

$$
\begin{aligned}
I_{C}=\int_{E} \int_{E(\boldsymbol{y}, \varepsilon)} V_{i j}(\boldsymbol{y}, \boldsymbol{x}) & n_{i}(\boldsymbol{y}) n_{j}(\boldsymbol{x}) \psi(\boldsymbol{y}) \varphi(\boldsymbol{x}) \mathrm{d} s_{x} \mathrm{~d} s_{y} \\
= & \int_{-1}^{1}\left\{\int_{\alpha(\varepsilon, u)}^{1} \frac{1}{v^{2}}\left[F^{(1)}(u, v)+F^{(2)}(u, v)\right] \mathrm{d} v\right\} \mathrm{d} u .
\end{aligned}
$$

It should be observed that this is an exact restatement of the original integral over $E \times E(\boldsymbol{y}, \varepsilon)$, with constant $\varepsilon$, in terms of a new pair of parametric coordinates. Of course, care has been taken to preserve the limiting process.

\subsection{The direct approach for coincident elements}

Following the direct algorithm for the evaluation of hypersingular integrals, the first two terms of expansion (41) are added and subtracted in (47) thus obtaining

$$
I_{C}=I_{0}+I_{1}+I_{2},
$$


having put

$$
\begin{aligned}
I_{0}= & \int_{-1}^{1}\left\{\int _ { \alpha ( \varepsilon , u ) } ^ { 1 } \left[F^{(1)}(u, v)+F^{(2)}(u, v)-2 F(u, 0)\right.\right. \\
& \left.\left.-\left(\frac{\partial F^{(1)}}{\partial v}(u, 0)+\frac{\partial F^{(2)}}{\partial v}(u, 0)\right) v\right] \frac{\mathrm{d} v}{v^{2}}\right\} \mathrm{d} u \\
I_{1}= & \int_{-1}^{1}\left(\frac{\partial F^{(1)}}{\partial v}(u, 0)+\frac{\partial F^{(2)}}{\partial v}(u, 0)\right) \int_{\alpha(\varepsilon, u)}^{1} \frac{\mathrm{d} v}{v} \mathrm{~d} u \\
I_{2}= & \int_{-1}^{1} 2 F(u, 0) \int_{\alpha(\varepsilon, u)}^{1} \frac{\mathrm{d} v}{v^{2}} \mathrm{~d} u,
\end{aligned}
$$

and where $F^{(1)}(u, 0)=F^{(2)}(u, 0)=F(u, 0)$.

As typical in the direct approach, the first double integral $I_{0}$ is now regular for $\varepsilon \rightarrow 0$, while the other potentially singular integrals $I_{1}$ and $I_{2}$ are trivial functions of $v$ and can always be integrated analytically, yielding

$$
\int_{\alpha(\varepsilon, u)}^{1} \frac{\mathrm{d} v}{v}=-\ln |\alpha(\varepsilon, u)|=\ln |2 a(u)|-\ln |\varepsilon|+O(\varepsilon)
$$

and

$$
\int_{\alpha(\varepsilon, u)}^{1} \frac{\mathrm{d} v}{v^{2}}=\frac{1}{\alpha(\varepsilon, u)}-1=\frac{2 a(u)}{\varepsilon}-\frac{u}{a(u)} \frac{\mathrm{d} a}{\mathrm{~d} u}(u)-1+O(\varepsilon),
$$

where the final expressions have been obtained simply by inserting expansions (46).

For the treatment of $I_{0}$ and $I_{1}$ we can take advantage of expression (43) for the sum of the first derivatives of $F$. Hence we have for $I_{0}$

$$
\begin{aligned}
& I_{0}=\int_{0}^{1}\left\{\int _ { - 1 } ^ { 1 } \left[F^{(1)}(u, v)+F^{(2)}(u, v)-2 F(u, 0)\right.\right. \\
&+\left.\left.2 \frac{\mathrm{d}}{\mathrm{d} u}(u F(u, 0)) v\right] \mathrm{~d} u\right\} \frac{\mathrm{d} v}{v^{2}}+O(\varepsilon) \\
&=\int_{0}^{1}\left\{\int_{-1}^{1}\left[F^{(1)}(u, v)+F^{(2)}(u, v)-2 F(u, 0)\right] \mathrm{d} u\right. \\
&+2[F(1,0)+F(-1,0)] v\} \frac{\mathrm{d} v}{v^{2}}+O(\varepsilon) .
\end{aligned}
$$

Similarly, for $I_{1}$ (using eqn. (49))

$$
\begin{aligned}
I_{1}= & -2 \int_{-1}^{1}\left\{\frac{\mathrm{d}}{\mathrm{d} u}(u F(u, 0)) \int_{\alpha(\varepsilon, u)}^{1} \frac{\mathrm{d} v}{v}\right\} \mathrm{d} u \\
= & 2 \int_{-1}^{1}\left\{\frac{\mathrm{d}}{\mathrm{d} u}(u F(u, 0))\right\}[\ln |\varepsilon|-\ln |2 a(u)|] \mathrm{d} u+O(\varepsilon) \\
= & 2\{\ln \varepsilon[F(1,0)+F(-1,0)]-F(1,0) \ln (2 a(1))-F(-1,0) \ln (2 a(-1))\} \\
& +2 \int_{-1}^{1}\left[u F(u, 0) \frac{1}{a(u)} \frac{\mathrm{d} a}{\mathrm{~d} u}(u)\right] \mathrm{d} u+O(\varepsilon) .
\end{aligned}
$$


As we can see, the final expressions in (51) and (52) do not require the explicit knowledge of the derivatives of $F$, but just of $F$ itself. This confirms what was anticipated in the comments to eqn. (43).

For $I_{2}$ the treatment is even simpler

$$
\begin{aligned}
I_{2} & =2 \int_{-1}^{1} F(u, 0)\left\{\int_{\alpha(\varepsilon, u)}^{1} \frac{\mathrm{d} v}{v^{2}}\right\} \mathrm{d} u \\
& =2 \int_{-1}^{1} F(u, 0)\left[\frac{2 a(u)}{\varepsilon}-\frac{u}{a(u)} \frac{\mathrm{d} a}{\mathrm{~d} u}(u)-1\right] \mathrm{d} u+O(\varepsilon)
\end{aligned}
$$

Combining equations (52) and (53) yields:

$$
\begin{array}{r}
I_{1}+I_{2}=\frac{1}{\varepsilon} \int_{-1}^{1} 4 F(u, 0) a(u) \mathrm{d} u+2 \ln \varepsilon[F(1,0)+F(-1,0)]-2 \int_{-1}^{1} F(u, 0) \mathrm{d} u \\
-2 F(1,0) \ln (2 a(1))-2 F(-1,0) \ln (2 a(-1))+O(\varepsilon) .
\end{array}
$$

It should be noted that in equation (54) there are an $O(\ln \varepsilon)$ term and an $O\left(\varepsilon^{-1}\right)$ term. Their cancellation is expected.

\section{Double integration over consecutive elements}

The evaluation of double integrals over consecutive elements follows a similar path, although it has some specific features. It is omitted here for brevity but can be found in [8].

\subsection{Cancellation of $\ln |\varepsilon|$ terms}

For any given weight function $\psi$ on $E$, the coincident, adjacent and transposed-adjacent types of integration all give rise to $\ln |\varepsilon|$ terms, whose magnitude depend only on the element geometry and density values at element $E$ endpoints. All these contributions add to zero provided the hypersingular kernels satisfy some conditions (which constitute indeed some of their properties) and in the algorithm the density function $\varphi$ is $C^{0}$ (continuous) across elements. It is worth remarking that the $C^{1, \alpha}$ smoothness required at $\varphi$ at $\boldsymbol{y}$ (cf. Eq. (13)) has no relevance here since $\boldsymbol{y}$ is never an endpoint for $E$. Therefore, $\varphi$ must be $C^{1, \alpha}$ strictly inside each boundary element (where it is usually $C^{\infty}$ ) and $C^{0}$ at both endpoints.

\section{Evaluation of free terms due to the hypersingularity}

Let us go back to the starting identity (16) for the Galerkin BEM. The following free term

$$
\int_{\Gamma} \psi(\boldsymbol{y}) \varphi(\boldsymbol{y})\left\{\int_{s_{\varepsilon}} V(\boldsymbol{y}, \boldsymbol{x}) \mathrm{d} s_{x}\right\} \mathrm{d} s_{y}
$$


appeared in equation (16) and needs further treatment to obtain its explicit expression. Once the boundary $\Gamma$ has been subdivided into boundary elements, one is led to compute the limiting form of the element-wise versions of (55)

$$
H=\int_{E} \psi(\boldsymbol{y}) \varphi(\boldsymbol{y})\left\{\int_{s_{\varepsilon}} V(\boldsymbol{y}, \boldsymbol{x}) \mathrm{d} s_{x}\right\} \mathrm{d} s_{y}
$$

Note that both $\psi$ and $\varphi$ are defined on $E$.

\subsection{Hypersingular free term final expression}

After several steps, as reported in [8], the final formula for the element-wise free term (56) is obtained

$$
H=H^{+}+H^{-}
$$

with

$$
\begin{aligned}
& H^{+}=\left\{\mathcal{H}_{i}(-\pi, 0,1)+\int_{0}^{\beta^{\prime}} \mathcal{H}_{i}(\theta, 0,1) \frac{\cos \left(\theta-\beta^{\prime}\right)}{\sin \beta^{\prime}} \mathrm{d} \theta\right\}\left[\psi \varphi n_{i}\right](1) \\
& H^{-}=-\left\{\mathcal{H}_{i}(0,0,-1)+\int_{0}^{\beta^{\prime \prime}} \mathcal{H}_{i}(\pi-\theta, 0,-1) \frac{\cos \left(\theta-\beta^{\prime \prime}\right)}{\sin \beta^{\prime \prime}} \mathrm{d} \theta\right\}\left[\psi \varphi n_{i}\right](-1)
\end{aligned}
$$

The finite free term (57) is specific to Galerkin BEM and apparently had never been detected before.

The free terms $H^{+}, H^{-}$are of coincident type in that they involve only $\varphi$ and $\psi$ on $E$, but also of adjacent type in that they depend on the angles $\beta^{\prime}, \beta^{\prime \prime}$ with neighbouring elements. It is useful to note that $\mathrm{H}^{+}+H^{-}=0$ if the tangent has interelement continuity. Since $\varphi$ must have interelement continuity, it is in practice more natural to treat $H^{+}, H^{-}$as adjacent terms. If we think of $\varphi$ as a continuous shape function, we see that it ought to be either $\varphi(-1)=0$ or $\varphi(1)=0$, which simplify the expression for the free term $H$. Moreover, if the support of a continuous weight function $\psi$ spans two boundary elements, we see that each side contributes a free term.

A short comment on the finite free terms just obtained is in order as they may look a bit unusual. They have never been detected before because in no other paper a limit process based on the vanishing neighbourhood approach has been used in conjunction with SGBEM. All formulations of SGBEM based on regularization of the kernels before performing the limit, like in $[11,12,13,14,15]$, do not provide free terms at all (and this is indeed one of their advantages). The same is true for the limit to the boundary method $[16,17,18]$. On the other hand, techniques based on the finite part idea $[19,20,21]$ completely overlook the free term evaluation, as the 'bump' associated to the vanishing neighbourhood is never taken into account.

The direct approach for the evaluation of singular integrals, here pursued and extended to SGBEM, is strongly based on a careful analysis of the limiting process, as the neighbourhood and the 'bump' around the singularity vanish, and free terms are therefore an essential part. It should also be considered that in the present paper the double integrations have been always dealt with as a whole, thus fully exploiting the features of the Galerkin BEM, and this aspect also affects the nature and the value of the free terms. 


\subsection{Evaluation of the free term $c(y)$}

Using the formalism introduced in this section, the free term $\boldsymbol{c}(\boldsymbol{y})$ defined by (15) becomes:

$$
\boldsymbol{c}(\boldsymbol{y})=\int_{-\pi}^{0}\{\mathcal{V}(\boldsymbol{e}(\omega ; \eta), 0, \boldsymbol{y}) \boldsymbol{e}(\omega ; \eta)-\mathcal{W}(\boldsymbol{e}(\omega ; \eta), 0, \boldsymbol{y}) \boldsymbol{n}(\boldsymbol{x})\} \mathrm{d} \omega
$$

where $\mathcal{W}(\boldsymbol{e}, r, \boldsymbol{y})=r W(\boldsymbol{y}, \boldsymbol{x})$ is the singular part of the strongly singular kernel $W(\boldsymbol{y}, \boldsymbol{x})$. Note that the integration bounds mean that $\Gamma$ is smooth at $\boldsymbol{y}$. This is the only case needed in connection with the weighted identity (16) as long as the irregular points on $\Gamma$ are isolated (e.g. a finite number of corners). Since $\boldsymbol{c}(\boldsymbol{y})$ are regular functions, the evaluation of the integral

$$
\int_{\Gamma} \psi(\boldsymbol{y}) \boldsymbol{\nabla} \varphi(\boldsymbol{y}) \cdot \boldsymbol{c}(\boldsymbol{y}) \mathrm{d} s_{y}
$$

in the identity (16) is a trivial task.

\section{Conclusions}

In this paper the extension of the direct approach to the evaluation of double hypersingular integrals, like those arising in the symmetric Galerkin BEM, has been outlined. Double integrals are considered as a whole through the introduction of suitable coordinate transformations in the two-dimensional space of intrinsic coordinates. As a result, the proposed procedure preserves the symmetry of the formulation after discretization, even when the numerical quadratures are affected by some errors. A complete treatment was presented in [8].

As typical in the direct method, the limiting process has been expressed in the parametric space and the free terms have been evaluated analytically. Cancellation of all potentially unbounded terms has been shown to occur if the density function $\varphi$ is continuous between elements. The analysis has also shown that somehow new free terms arise.

\section{References}

[1] F. Rizzo, An integral equation approach to boundary value problems of classical elastostatics, Quarterly of Applied Mathematics 25 (1967) 83-95.

[2] M. Guiggiani, P. Casalini, Direct computation of Cauchy principal value integrals in advanced boundary elements, Int. J. Num. Meth. in Eng. 24 (1987) 1711-1716.

[3] M. Guiggiani, A. Gigante, A general algorithm for multidimensional Cauchy principal value integrals in the boundary element method., ASME J. Appl. Mech. 57 (1990) 906-915. 
[4] M. Guiggiani, G. Krishnasamy, T. J. Rudolphi, F. J. Rizzo, A general algorithm for the numerical solution of hypersingular boundary integral equations., ASME J. Appl. Mech. 59 (1992) 604-614.

[5] M. Guiggiani, Hypersingular formulation for boundary stress evaluation., Engng. Anal. with Bound. Elem. 14 (1994) 169-179.

[6] M. Guiggiani, Hypersingular boundary integral equations have an additional free term., Comp. Mech. 16 (1995) 245-248.

[7] A. Frangi, M. Guiggiani, A direct approach for boundary integral equations with high-order singularities., Int. J. Num. Meth. in Eng. 49 (2000) 871-898.

[8] M. Bonnet, M. Guiggiani, Direct evaluation of double singular integrals and new free terms in 2D (symmetric) Galerkin BEM, Comp. Meth. in Appl. Mech. Engng. 192 (2003) 2565-2596.

[9] S. Sirtori, General stress analysis method by means of integral equations and boundary elements., Meccanica 14 (1979) 210-218.

[10] G. Maier, C. Polizzotto, A Galerkin approach to boundary element elastoplastic analysis., Comp. Meth. in Appl. Mech. Engng. 60 (1987) 175-194.

[11] J. H. Kane, C. Balakrishna, Symmetric Galerkin boundary formulations employing curved elements., Int. J. Num. Meth. in Eng. 36 (1993) 2157-2187.

[12] M. Bonnet, Regularized direct and indirect symmetric variational BIE formulations for three-dimensional elasticity., Engng. Anal. with Bound. Elem. 15 (1995) 93-102.

[13] S. Sirtori, G. Maier, G. Novati, S. Miccoli, A Galerkin symmetric boundary element method in elasticity: formulation and implementation., Int. J. Num. Meth. in Eng. 35 (1992) 255-282.

[14] A. Frangi, G. Novati, Symmetric BE method in two-dimensional elasticity: evaluation of double integrals for curved elements., Comp. Mech. 19 (1996) 58-68.

[15] A. Frangi, M. Bonnet, A Galerkin symmetric and direct BIE method for Kirchhoff elastic plates: formulation and implementation., Int. J. Num. Meth. in Eng. 41 (1998) 337-369.

[16] C. Balakrishna, L. J. Gray, J. H. Kane, Efficient analytical integration of symmetric Galerkin boundary integrals over curved elements: thermal conduction formulation., Comp. Meth. in Appl. Mech. Engng. 111 (1994) 335-355.

[17] C. Balakrishna, L. J. Gray, J. H. Kane, Efficient analytical integration of symmetric Galerkin boundary integrals over curved elements: elasticity formulation., Comp. Meth. in Appl. Mech. Engng. 117 (1994) 157-179. 
[18] L. Gray, Evaluation of singular and hypersingular Galerkin integrals: direct limits and symbolic computation., in: V. Sladek, J. Sladek (Eds.), Singular Integrals in Boundary Element Methods, Comp. Mech. Publ., Southampton, 1998, Ch. 2, pp. 45-84.

[19] A. Aimi, M. Diligenti, G. Monegato, New numerical integration schemes for applications of Galerkin BEM to 2-D problems., Int. J. Num. Meth. in Eng. 40 (1997) 1977-1999.

[20] A. Aimi, M. Diligenti, G. Monegato, Numerical integration schemes for the BEM solution of hypersingular integral equations., Int. J. Num. Meth. in Eng. 45 (1999) 1807-1830.

[21] A. Carini, M. Diligenti, P. Maranesi, M. Zanella, Analytical integrations for two-dimensional elastic analysis by the symmetric Galerkin boundary element method., Comp. Mech. 23 (1999) 308-323.

[22] W. Hackbush, S. A. Sauter, On the efficient use of the Galerkin method to solve Fredholm integral equation., Applications of Mathematics 38 (1993) 301-322.

[23] T. von Petersdorff, C. Schwab, Fully discrete multiscale Galerkin BEM, Tech. Rep. 95-08, ETH, Zurich, Switzerland (1995).

[24] S. A. Sauter, C. Schwab, Quadrature for $h p$-Galerkin BEM in $R^{3}$., Numerische Mathematik 78 (1997) 211-258.

[25] S. Erichsen, S. A. Sauter, Efficient automatic quadrature in 3-D Galerkin BEM., Comp. Meth. in Appl. Mech. Engng. 157 (1998) 215-224.

[26] H. Andrä, E. Schnack, Integration of singular Galerkin-type boundary element integrals for 3D elasticity problems., Numerische Mathematik 76 (1997) 143165 .

[27] H. Andrä, Integration of singular integrals for the Galerkin-type boundary element method in 3D elasticity., Comp. Meth. in Appl. Mech. Engng. 157 (1998) 239-249. 